\title{
Linear Stability Analysis of Thermal Convection in an Infinitely Long Vertical Rectangular Enclosure in the Presence of a Uniform Horizontal Magnetic Field
}

\author{
Takashi Kitaura and Toshio Tagawa \\ Tokyo Metropolitan University, 6-6, Asahigaoka, Hino-shi 191-0065, Japan \\ Correspondence should be addressed to Toshio Tagawa; tagawa-toshio@tmu.ac.jp
}

Received 23 January 2014; Accepted 2 April 2014; Published 29 April 2014

Academic Editor: Robert Spall

Copyright (C) 2014 T. Kitaura and T. Tagawa. This is an open access article distributed under the Creative Commons Attribution License, which permits unrestricted use, distribution, and reproduction in any medium, provided the original work is properly cited.

Stability of thermal convection in an infinitely long vertical channel in the presence of a uniform horizontal magnetic field applied in the direction parallel to the hot and cold walls was numerically studied. First, in order to confirm accuracy of the present numerical code, the one-dimensional computations without the effect of magnetic field were computed and they agreed with a previous study quantitatively for various values of the Prandtl number. Then, linear stability analysis for the thermal convection flow in a square horizontal cross section under the magnetic field was carried out for the case of $\operatorname{Pr}=0.025$. The thermal convection flow was once destabilized at certain low Hartmann numbers, and it was stabilized at high Hartmann numbers.

\section{Introduction}

Nuclear fusion energy has received considerable attention as one of the environment-friendly in modern society. Heading towards implementation of the use of nuclear fusion energy, experimental facility called ITER (International Thermonuclear Experimental Reactor) is currently under construction in France. ITER has a toroidal shape like a donut, in which the high-temperature plasma enough to induce nuclear fusion reaction is controlled by both operation of magnetic field generated by the superconducting coils disposed around the plasma and the imposed electric current in the plasma [1]. Blankets located close to the plasma side play an important role for cooling, shielding neutrons, and fuel production.

Fusion reactor blanket can be classified into the solid blanket using a solid compound of lithium as fuel production material, or liquid blanket using liquid lithium. Above all, liquid blanket has the advantage of being of relatively simple structure [2], but, on the other hand, it has a serious problem called the MHD pressure loss [3]. The MHD pressure loss obstructs convection of liquid metal as a coolant and it depends on the direction of the magnetic field and the electric conductivity of the wall. To elucidate that problem, researches on thermal convection under the electromagnetic force have been actively conducted not only for the application of fusion reactor blankets but also for crystal growth such as the horizontal Bridgman method.

The effect of the direction of uniform magnetic field on the natural convection in a cubic enclosure heated from a vertical wall and cooled from an opposing vertical wall was numerically studied by Ozoe and Okada [5]. They showed that the horizontal magnetic field parallel to the hot and cold walls ( $Y$-directional magnetic field) has much less influence on the damping of natural convection than the two other directions of magnetic field ( $X$ - or $Z$-directional magnetic field). This finding was confirmed with an experiment using $30 \mathrm{~mm} \times 30 \mathrm{~mm} \times 30 \mathrm{~mm}$ cubical box filled with molten gallium by Okada and Ozoe [6]. They noticed the heat transfer rate of natural convection under the $Y$-directional magnetic field indicated not only weak damping of heat transfer rate at high magnetic fields but also slight enhancement of heat transfer rate at low magnetic fields. However, they were not convinced by this enhancement of heat transfer. 
After several years, Tagawa and Ozoe $(1997,1998)[7,8]$ carried out both three-dimensional computations with the higher Rayleigh numbers than those by Ozoe and Okada and the molten gallium experiment using $64 \mathrm{~mm} \times 64 \mathrm{~mm} \times$ $64 \mathrm{~mm}$ cubical box for the $Y$-directional magnetic field in order to confirm the existence of enhancement of heat transfer rate at low magnetic fields. Due to the exploration of the larger Rayleigh numbers, both the numerical and experimental results showed the enhancement of heat transfer rate (the Nusselt number) when the uniform magnetic field was applied in the $Y$-direction.

The enhancement of Nusselt number in the presence of low magnetic fields has been reported by several other groups. Authié et al. [9] carried out both three-dimensional computations and the corresponding experiment using mercury for the buoyant convection in a long vertical enclosure. Their experiments indicated that the Nusselt number takes its maximum around the Hartmann number 200-250 for the values of the Grashof number from $3 \times 10^{7}$ to $1.5 \times 10^{8}$. Burr et al. [10] carried out the natural convection for the similar configuration under a horizontal magnetic field perpendicular to both the gravity and the applied heat flux. They showed that the Nusselt numbers increased from the value of natural convection without the magnetic field in the range of the Hartmann number $100<\mathrm{Ha}<225$ with a tendency of higher Hartmann numbers at larger heat fluxes. Zhang et al. [11] carried out an experimental study on the flow structure of a bubble-driven liquid-metal jet in a horizontal magnetic field. They concluded that the application of a moderate magnetic field destabilizes the global flow and gives rise to transient, oscillating flow patterns with predominant frequencies. The electric conducting fluid motion is usually damped by the use of static magnetic field. The above-mentioned literatures concern the enhancement of natural convection heat transfer or destabilizing effect of the electric conducting fluid flow at low Hartmann numbers in the presence of static magnetic field. This interesting finding could be related to the flow instability and/or transition.

Fujimura and Nagata (1998) [12] studied instability of the natural convection in an infinitely long vertical rectangular channel in the presence of a uniform horizontal magnetic field by two-dimensional analysis. Kakutani (1964) [13] studied the hydromagnetic stability on the plane laminar flow between the parallel plates in the presence of a transverse magnetic field. The plane Couette flow is destabilized by the magnetic field in the range of Hartmann number $3.91<\mathrm{Ha}<$ 5.4.

The purpose of this research is to clarify the stability of parallel flow of thermal convection in an infinitely long vertical rectangular channel in the presence of a horizontal magnetic field.

\section{Numerical Model}

The schematic model considered in this study is shown in Figure 1. The fluid treated in this research is assumed to be an incompressible Newtonian fluid, and the Boussinesq approximation is employed. The boundary conditions are

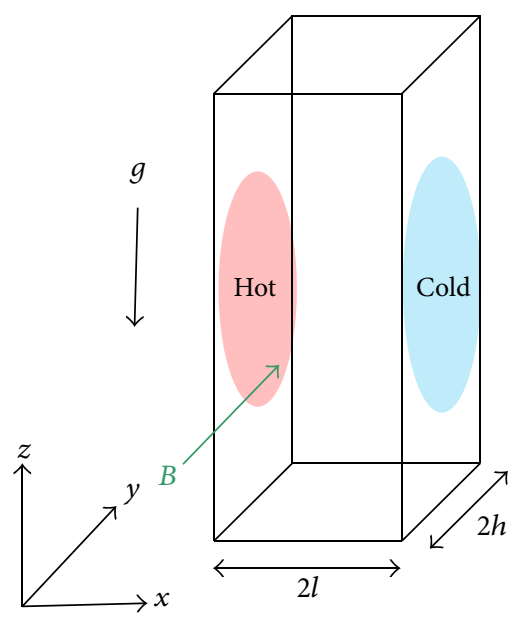

Figure 1: Schematic model considered.

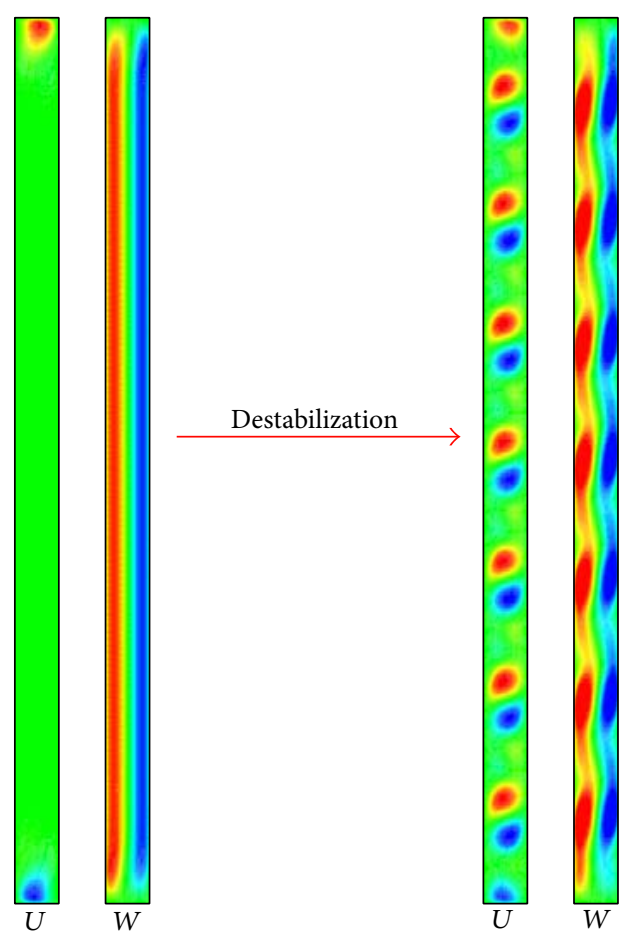

FIgURE 2: An example of destabilization of the thermal convection in a long vertical rectangular channel at $\mathrm{Pr}=0.025$ and $\mathrm{Gr}=900.0$.

the no-slip condition for all walls and the heated and cooled walls are isothermal and the other two walls are the thermally insulated. Governing equations are the equations of continuity, the momentum, the energy, Ohm's law, and the conservation of electric charge. We used a finite difference method with a staggered mesh system. By introducing the time derivative term into the governing equations, we obtain approximated solutions under discretization using a secondorder or fourth-order accurate central difference method, together with the use of HS-MAC method for the solution of the Poisson equations of the pressure and the electric potential. 


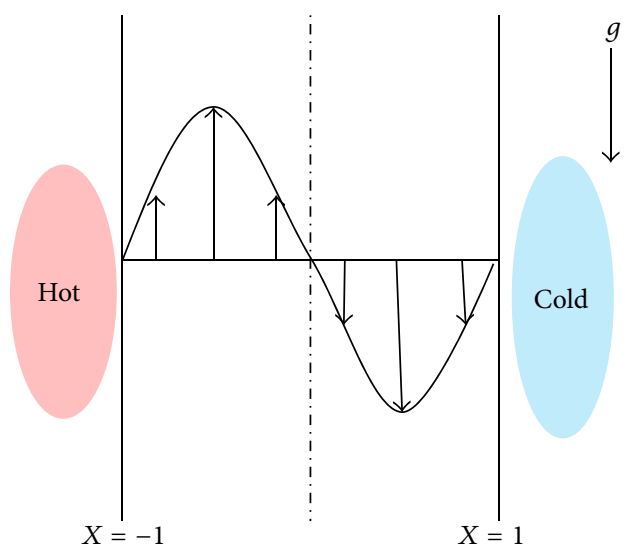

Figure 3: Simplified calculation model $(A=h / l \rightarrow \infty)$.

TABLE 1: Comparisons of the critical Grashof number and the critical wavenumber between a reference and the present research at $\operatorname{Pr}=$ 0.67 and 7.5 .

\begin{tabular}{lcccc}
\hline Method & \multicolumn{2}{c}{ Reference [4] } & \multicolumn{2}{c}{ Present } \\
& \multicolumn{2}{c}{$\begin{array}{c}\text { Chebyshev polynomials } \\
\text { expansion }\end{array}$} & $\begin{array}{c}\text { Fourth-order accurate } \\
\text { central difference } \\
\text { method with 101 grids }\end{array}$ \\
\hline $\operatorname{Pr}$ & 0.67 & 7.5 & 0.67 & 7.5 \\
$k_{c}$ & 1.40417 & 1.38334 & 1.40419 & 1.38349 \\
$\mathrm{Gr}_{c}$ & 503.293 & 491.900 & 503.305 & 491.829 \\
\hline
\end{tabular}

The instability of the convection of the infinitely long vertical rectangular channel is investigated by using the Grashof number, which is a dimensionless number representing the magnitude of the influence of the inertial and buoyancy forces. Figure 2 shows how the flow of a two-dimensional vertical rectangular channel becomes unstable. When the Grashof number exceeds a certain value, the flow structure is divided into a number of cells. In this study, the destabilized flow in the three-dimensional vertical rectangular channel is assumed to be periodic flow in the $Z$-axis direction, and then we perform the computation with applying a uniform horizontal magnetic field perpendicular to the temperature gradient.

\section{Verification of the Numerical Code}

As the verification of the numerical code, preliminary computations were performed for the model of the aspect ratio $(h / l \rightarrow \infty)$ without magnetic field, and those results were compared with previous results [4]. The present model and the numerical results are described in Figures 3 and 4, respectively. Figure 4 describes the result performed with the number of grids 101 using the discretization of a fourth-order accurate central difference method.

It is noted that this graph shows result of the case of the standing wave disturbance. It is known that for the lower Prandtl number $(\operatorname{Pr}<12.45)$ [14], the neutral Grashof number in the case of the standing wave disturbance is lower than that in the case of the traveling wave disturbance.
Table 1 shows the numerical results for comparison of the critical Grashof number and the critical wavenumber when air $(\operatorname{Pr}=0.67)$ and water $(\operatorname{Pr}=7.5)$ are assumed as test fluids. The agreement between the reference's result and the present result is negligibly small.

\section{Dimensionless Equations}

The flow instability in a long vertical enclosure in the presence of a magnetic field applied in the $Y$-direction is investigated here. In this study, the destabilized flow is assumed to be periodic and stationary in the $Z$-axis direction, and then the numerical analyses were performed within a horizontal cross section. Due to the computational resources, we focus on the case that the aspect ratio of the horizontal cross section is unity and the Prandtl number is 0.025 , which is a typical value of liquid metals.

The dimensionless governing equations are shown below:

$$
\begin{gathered}
\frac{\partial \mathbf{V}}{\partial \tau}+(\mathbf{V} \cdot \nabla) \mathbf{V}=-\nabla P+\frac{1}{\mathrm{Gr}} \nabla^{2} \mathbf{V}+\frac{1}{\mathrm{Gr}} \Theta \mathbf{e}_{z}+\frac{\mathrm{Ha}^{2}}{\mathrm{Gr}} \mathbf{J} \times \mathbf{e}_{B_{0}}, \\
\frac{\partial \Theta}{\partial \tau}+\mathbf{V} \cdot \nabla \Theta=\frac{1}{\mathrm{GrPr}} \nabla^{2} \Theta, \\
\nabla \cdot \mathbf{V}=0, \quad \nabla \cdot \mathbf{J}=0, \\
\mathbf{J}=-\nabla \Phi+\mathbf{V} \times \mathbf{e}_{B_{0}},
\end{gathered}
$$

The dimensionless variables are defined as follows:

$$
\begin{aligned}
\tau & =\frac{t}{l^{2} /(\mathrm{Gr} v)}, \quad \mathbf{X}=\frac{\mathbf{x}}{l}, \quad \mathbf{V}=\frac{\mathbf{v}}{\mathrm{Gr} v / l}, \\
\Theta & =\frac{T-T_{0}}{\Delta T}, \quad P=\frac{p}{\rho_{0} \mathrm{Gr}^{2} \nu^{2} / l^{2}}, \\
\mathbf{J} & =\frac{\mathbf{j}}{\mathrm{Gr} \nu B_{0}}, \quad \Phi=\frac{\phi}{\mathrm{Gr} \sigma_{e} \nu B_{0} / l}, \quad \mathrm{Gr}=\frac{g \beta \Delta T l^{3}}{\nu^{2}}, \\
\operatorname{Pr} & =\frac{\nu}{\alpha}, \quad \mathrm{Ha}=B_{0} l \sqrt{\frac{\sigma_{e}}{\rho_{0} \nu}}, \quad A=\frac{h}{l} .
\end{aligned}
$$

Since we limit ourselves to the case that the uniform magnetic field is applied in the $Y$-direction, it holds that $\mathbf{e}_{B_{0}}=\mathbf{e}_{y}$.

\section{Basic Flow}

First, the flow of the basic state is computed with the assumption that the rectangular enclosure is long enough to neglect recirculation area near the top and the bottom walls. Therefore, the basic state of temperature field is in heat conduction. Since the Prandtl and Grashof numbers are included in the scaling, the dimensionless basic flow is independent of the Grashof and Prandtl numbers. Dimensionless governing equations used in the computation are shown as follows. 


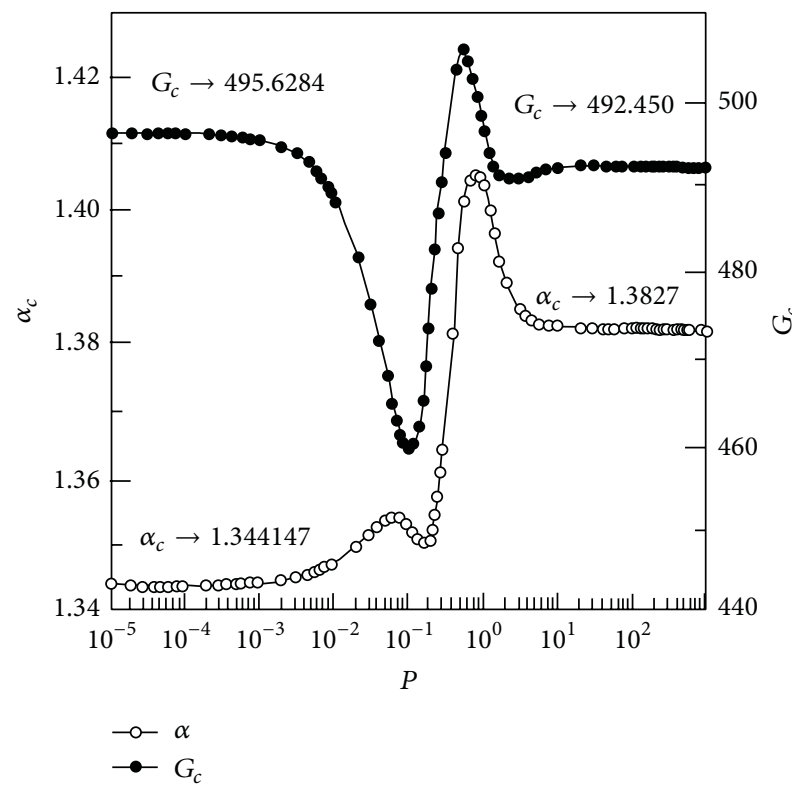

(a) Reference [4]

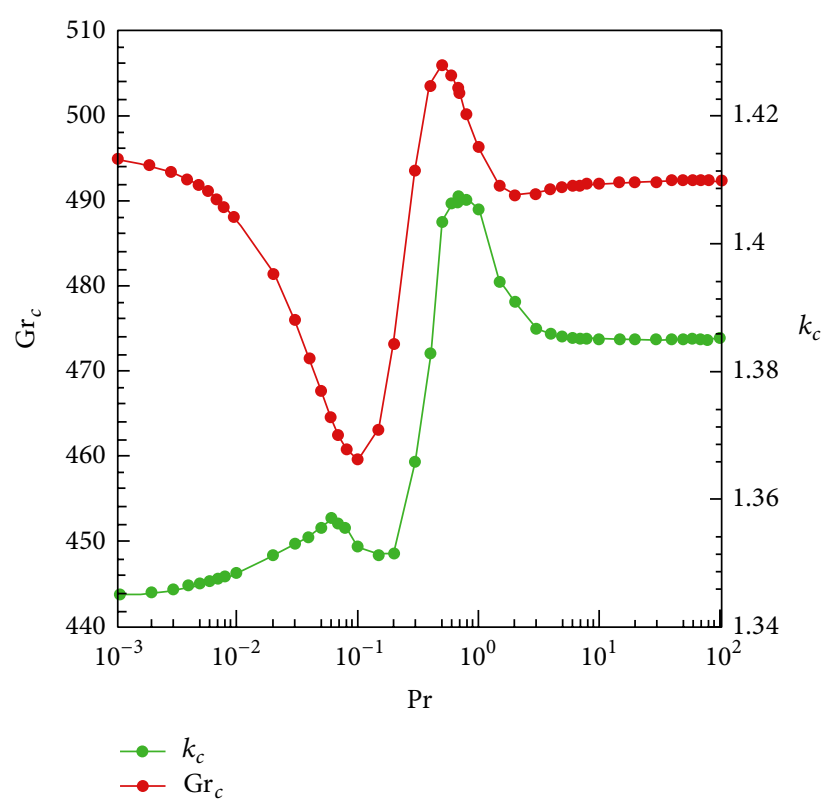

(b) Present result

FIgURE 4: Variations of the critical Grashof number and the critical wavenumber for the wide range of the Prandtl number. (a) is referred from a previous study [4] and (b) indicates the present result.

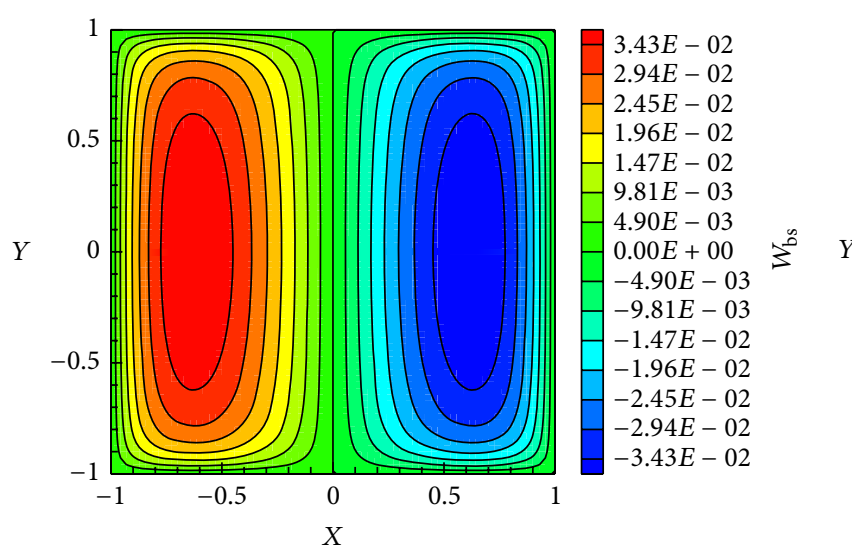

(a)

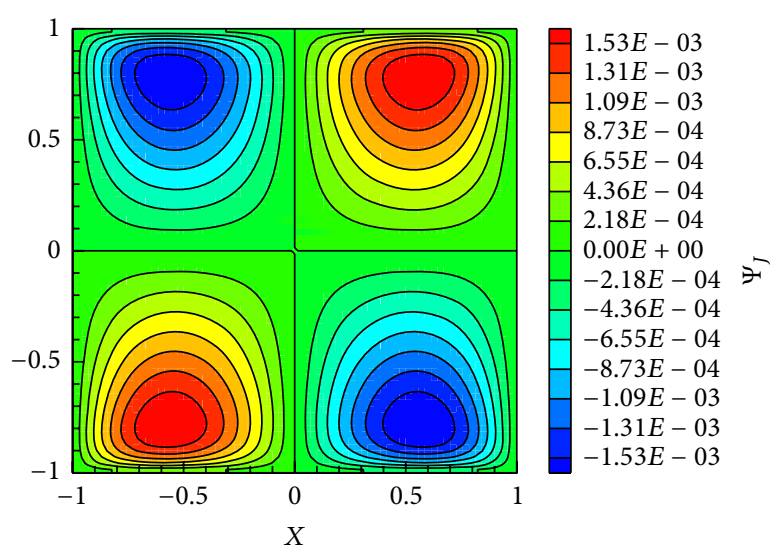

(b)

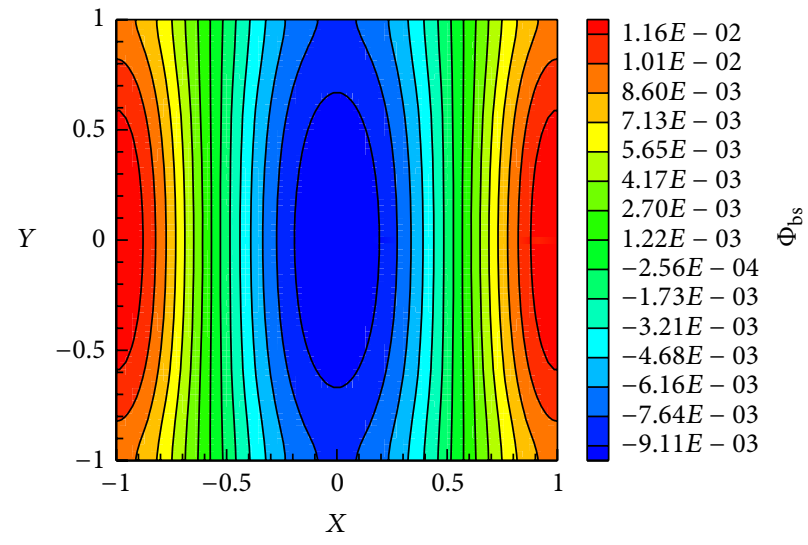

(c)

FIGURE 5: Contour maps of the basic flow at $\mathrm{Ha}=10.0$ : (a) vertical velocity $W_{\mathrm{bs}}$, (b) stream line of electric current $\Psi_{J}$, and (c) electric potential $\Phi_{\text {bs }}$. 
In this study, the aspect ratio of the horizontal cross section is limited to $A=h / l=1$ :

$$
\begin{gathered}
\frac{\partial^{2} W_{\mathrm{bs}}}{\partial X^{2}}+\frac{\partial^{2} W_{\mathrm{bs}}}{\partial Y^{2}}+\Theta_{\mathrm{bs}}+\mathrm{Ha}^{2} J x_{\mathrm{bs}}=0, \\
\Theta_{\mathrm{bs}}=-X \\
\frac{\partial J x_{\mathrm{bs}}}{\partial X}+\frac{\partial J y_{\mathrm{bs}}}{\partial Y}=0, \\
J x_{\mathrm{bs}}=-\frac{\partial \Phi_{\mathrm{bs}}}{\partial X}-W_{\mathrm{bs}} \\
J y_{\mathrm{bs}}=-\frac{\partial \Phi_{\mathrm{bs}}}{\partial Y} .
\end{gathered}
$$

Here, the subscript character bs represents the basic state. The vertical component of velocity $W$, the streamlines of electric current density $\Psi_{j}$, and the electric potential $\Phi$ of the basic state at the Hartmann number $\mathrm{Ha}=10.0$ are depicted in Figure 5.

\section{Linear Stability Analysis}

Disturbance equations are derived for investigating the flow instability. First of all, velocity, pressure, temperature, electric potential, and electric current density are represented with the sum of the solution of the basic state and the infinitesimal disturbances [15]:

$$
\begin{array}{ll}
\mathbf{v}=\mathbf{v}_{\mathrm{bs}}+\mathbf{v}^{\prime}, & p=p_{\mathrm{bs}}+p^{\prime}, \\
T=T_{\mathrm{bs}}+T^{\prime}, & \phi=\phi_{\mathrm{bs}}+\phi^{\prime}, \\
\mathbf{j}=\mathbf{j}_{\mathrm{bs}}+\mathbf{j}^{\prime} . &
\end{array}
$$

We assume that the infinitesimal disturbances are represented by the normal mode as follows:

$$
\left(\mathbf{v}^{\prime}, p^{\prime}, T^{\prime}, \phi^{\prime}, \mathbf{j}^{\prime}\right)=(\widetilde{\mathbf{v}}, \widetilde{p}, \widetilde{T}, \widetilde{\phi}, \widetilde{\mathbf{j}}) \exp (\mathrm{i} a z+\lambda t)
$$

Note that $i$ is the imaginary unit, $\lambda$ is the complex growth rate which is a complex number, and $a$ is the wavenumber which is a real number here. The dimensionless equations obtained by substituting (4) and (5) to (1) are shown below. It is known that the natural convection flow in a vertical slot becomes unstable for standing wave disturbance rather than for travelling wave disturbance in the range of
TABLE 2: Variation of the neutral Grashof number for several cases of number of grids at $\mathrm{Pr}=0.025, k=0.83$, and $\mathrm{Ha}=5.0$.

\begin{tabular}{lcc}
\hline Number of meshes & 2nd-order & 4th-order \\
\hline $30 \times 30$ & 2280.63 & 2191.22 \\
$50 \times 50$ & 2177.64 & 2152.50 \\
$70 \times 70$ & 2153.61 & 2142.76 \\
\hline
\end{tabular}

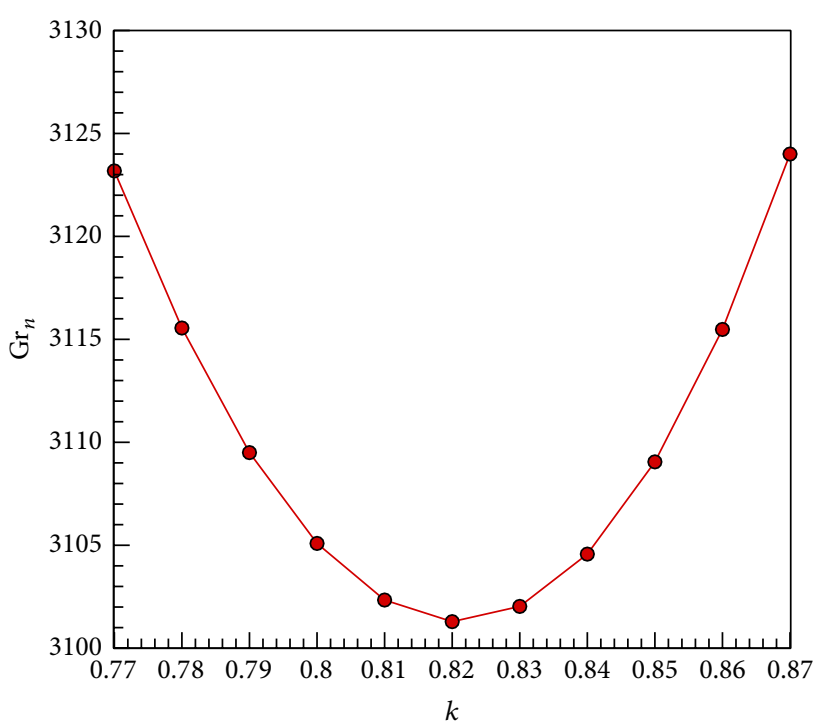

FIGURE 6: Neutral stability curve of the Grashof number at $\mathrm{Pr}=$ 0.025 and $\mathrm{Ha}=10.0$.

$\operatorname{Pr}<12.45$ [14]. Therefore, we assumed that the neutral stable state of the flow of $\operatorname{Pr}=0.025$ is taken as $\lambda=0$ :

$$
\begin{gathered}
\frac{\partial \widetilde{U}}{\partial X}+\frac{\partial \widetilde{V}}{\partial Y}+i k \widetilde{W}=0, \\
i W_{\mathrm{bs}} k \widetilde{U}=-\frac{\partial \widetilde{P}}{\partial X}+\frac{1}{\mathrm{Gr}}\left(\frac{\partial^{2}}{\partial X^{2}}+\frac{\partial^{2}}{\partial Y^{2}}-k^{2}\right) \widetilde{U}+\frac{\mathrm{Ha}^{2}}{\mathrm{Gr}}(-\widetilde{J} z), \\
i W_{\mathrm{bs}} k \widetilde{V}=-\frac{\partial \widetilde{P}}{\partial Y}+\frac{1}{\mathrm{Gr}}\left(\frac{\partial^{2}}{\partial X^{2}}+\frac{\partial^{2}}{\partial Y^{2}}-k^{2}\right) \widetilde{V}, \\
\widetilde{U} \frac{\partial W_{\mathrm{bs}}}{\partial X}+\widetilde{V} \frac{\partial W_{\mathrm{bs}}}{\partial Y}+i W_{\mathrm{bs}} k \widetilde{W} \\
=-i k \widetilde{P}+\frac{1}{\mathrm{Gr}}\left(\frac{\partial^{2}}{\partial X^{2}}+\frac{\partial^{2}}{\partial Y^{2}}-k^{2}\right) \widetilde{W}+\frac{1}{\mathrm{Gr}} \widetilde{\Theta}+\frac{\mathrm{Ha}^{2}}{\mathrm{Gr}} \widetilde{J} x, \\
\frac{\partial \widetilde{J} x}{\partial X}+\frac{\partial \widetilde{J} y}{\partial Y}+i k \widetilde{J} z=0, \quad \widetilde{J} x=-\frac{\partial \widetilde{\Phi}}{\partial X}-\widetilde{W}, \\
\widetilde{J} y=-\frac{\partial \widetilde{\Phi}}{\partial Y}, \quad \widetilde{J} z=-i k \widetilde{\Phi}+\widetilde{U} .
\end{gathered}
$$

Table 2 shows variation of the critical Grashof number on the number of grids at the dimensionless wavenumber $k=$ 0.83 and Hartmann number $\mathrm{Ha}=5.0$ when discretized with 


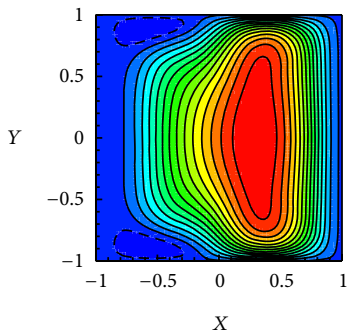

(a)

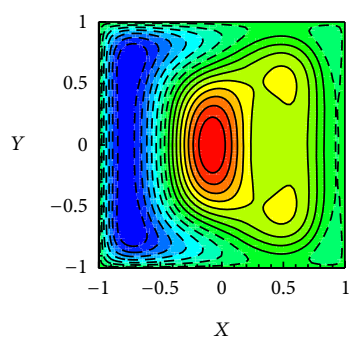

(e)

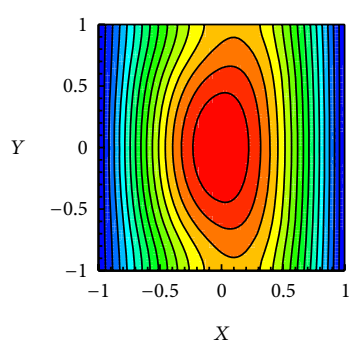

(i)

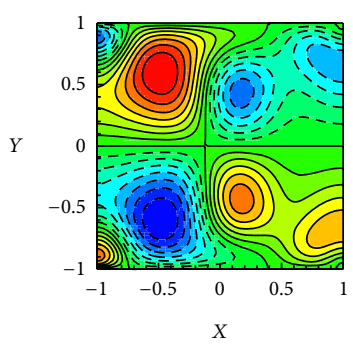

(m)

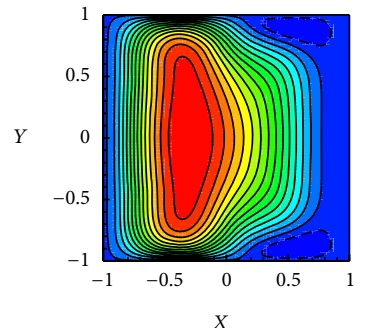

(b)

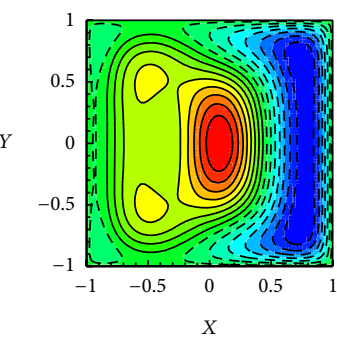

(f)

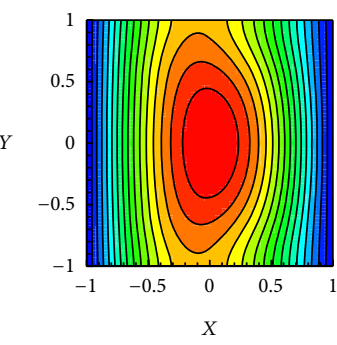

(j)

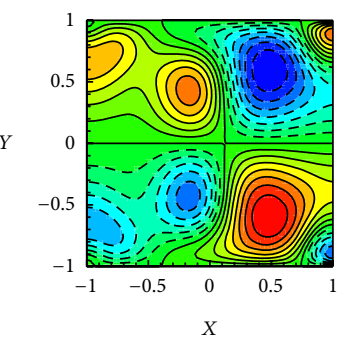

(n)

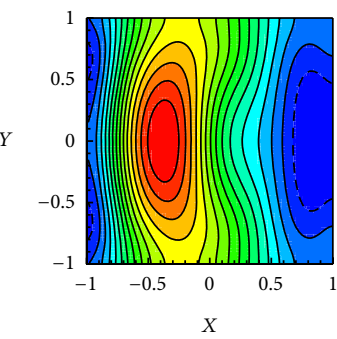

(q)

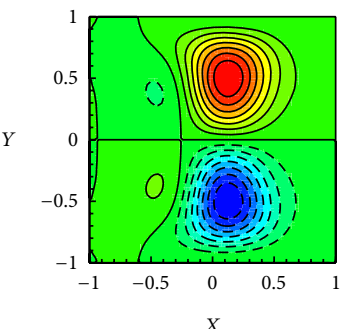

(c)

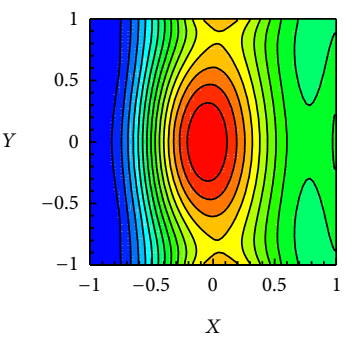

(g)

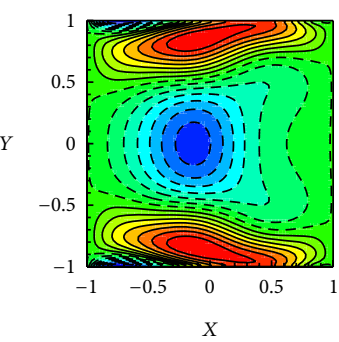

(k)

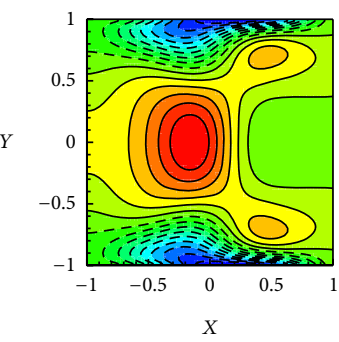

(o)

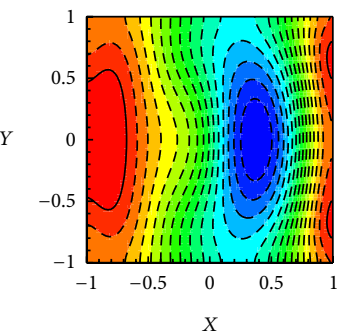

(r)

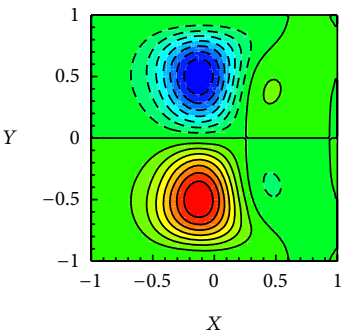

(d)

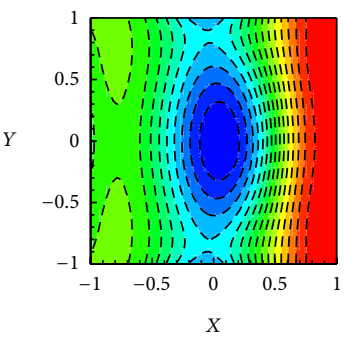

(h)

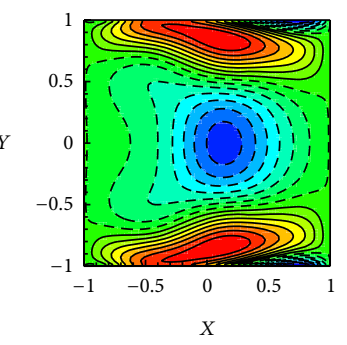

(1)

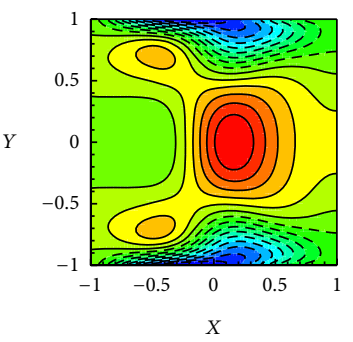

(p)

FIGURE 7: Contour maps of the characteristic function at a marginal state at $\operatorname{Pr}=0.025, k=0.83, \mathrm{Gr}=2152.5$, and $\mathrm{Ha}=5.0$ : (a) real part of $\widetilde{U}$, (b) imaginary part of $\widetilde{U}$, (c) real part of $\widetilde{V}$, (d) imaginary part of $\widetilde{V}$, (e) real part of $\widetilde{W}$, (f) imaginary part of $\widetilde{W}$, (g) real part of $\widetilde{P}$, (h) imaginary part of $\widetilde{P}$, (i) real part of $\widetilde{\Theta},(\mathrm{j})$ imaginary part of $\widetilde{\Theta},(\mathrm{k})$ real part of $J \widetilde{x},(\mathrm{l})$ imaginary part of $J \widetilde{x},(\mathrm{~m})$ real part of $J \widetilde{y}$, (n) imaginary part of $J \widetilde{y},(\mathrm{o})$ real part of $J \widetilde{z}$, (p) imaginary part of $J \widetilde{z}$, (q) real part of $\widetilde{\Phi}$, and (r) imaginary part of $\widetilde{\Phi}$. 


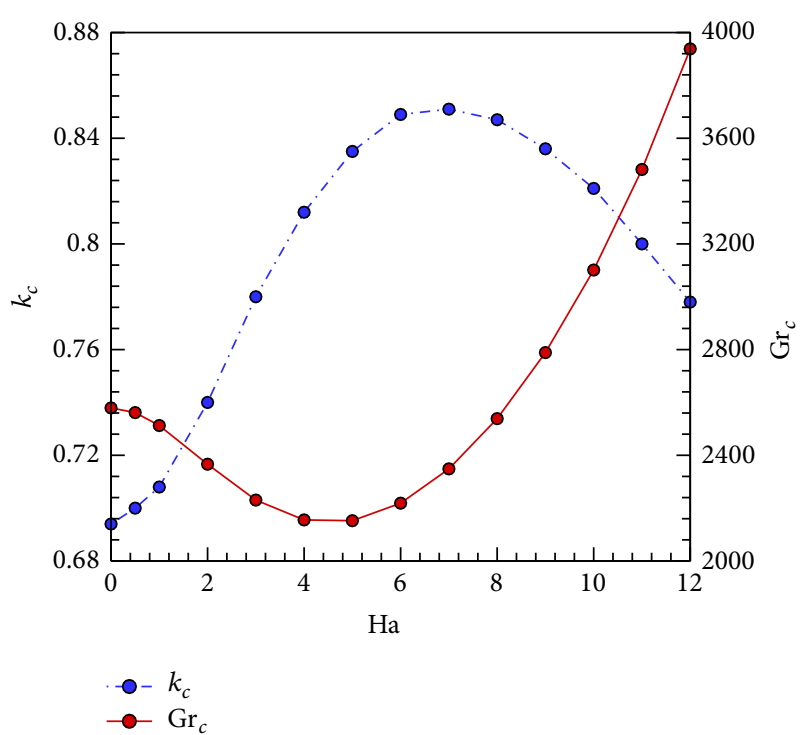

FIGURE 8: Variation of the critical Grashof number and the critical wavenumber against the Hartmann number at $\operatorname{Pr}=0.025$.

a second-order or a fourth-order accurate central difference. Considering the result of Table 2 and the computational time, we employed $70 \times 70$ mesh number with the second-order accurate central difference. For example, the neutral stability curve of the Grashof number at Hartmann number $\mathrm{Ha}=10.0$ is depicted in Figure 6. The critical Grashof numbers at the wavenumber 0.821 with mesh size $70 \times 70,90 \times 90$ and $120 \times$ 120 take the value of 3101,3109, and 3114, respectively. So the dependency of the mesh size on the result is not so significant.

Characteristic functions for various variables at a neutral state are illustrated in Figure 7. The pressure and the potential distribution have point symmetry and have reverse sign between the real and the imaginary parts with respect to $X=$ 0 and $Y=0$. On the other hand, the velocity, temperature, and electric current density distribution have point symmetry with respect to $X=0$ and $Y=0$.

The critical Grashof number and the wavenumber plotted against the Hartmann number up to 12 are shown in Figure 8. Concerning the application of a horizontal Bridgeman crystal growth, Lyubimov et al. [16] studied the stability of thermal convection in a rectangular cross section of an oblong channel. They studied two cases of computation, the magnetic field applied in the horizontal direction or in the vertical direction with changing the Prandtl number and the aspect ratio of cross section. It should be noted that the present study could be compared with the study of Lyubimov et al. at the case of zero value of Prandtl number $(\operatorname{Pr}=0)$. In the configuration of horizontal Bridgeman crystal growth, the plane-parallel flow does not exist in nonzero values of Prandtl number, but in the present study the plane-parallel flow always exists irrespective of Prandtl number since the basic thermal state is independent of Prandtl number. According to the results of horizontal magnetic field by Lyubimov et al., the critical Grashof number takes its minimum at a certain low Hartmann number and the critical wavenumber takes its maximum at another low Hartmann number. This tendency is quite coincident with the finding of the present study. This indicates that a moderate magnetic field destabilizes the natural convection of plane-parallel flow but a strong magnetic field stabilizes the convection.

\section{Conclusion}

In this study, we studied the linear stability of thermal convection in an infinitely long vertical rectangular channel in the presence of a uniform horizontal magnetic field. We obtained some findings. First, the characteristic function of the pressure and the electric potential distribution have point symmetry and have reverse sign between the real and the imaginary parts with respect to the center of cross section. On the other hand, the velocity, temperature, and electric current density distribution have point symmetry with respect to the center of cross section. Second, except in the range of the Hartmann number of $0<\mathrm{Ha}<8$, increase in the Hartmann number leads to well stabilization. And the critical Grashof number takes a minimum value at the Hartmann number of about $\mathrm{Ha}=4.5$. Third, the critical wavenumber increases at a low Hartmann number but then decreases for further increase in the Hartmann number.

\section{Nomenclature}

a: Wavenumber $[\mathrm{rad} / \mathrm{m}]$

A: $\quad$ Aspect ratio $(=h / l)$

$B_{0}: \quad$ Applied magnetic field $[\mathrm{T}]$

$\mathbf{e}_{B_{0}}$ : Unit vector (direction of $B_{0}$ )

$\mathbf{e}_{y}$ : Unit vector (direction of $y$-axis)

$\mathbf{e}_{z}$ : Unit vector (direction of $z$-axis)

Gr: Grashof number

$g: \quad$ Gravitational acceleration $\left[\mathrm{m} / \mathrm{s}^{2}\right]$

$h$ : $\quad$ Duct width in the $y$-axis [m]

Ha: Hartmann number

$i$ Imaginary unit

j: $\quad$ Electric current density vector $\left[\mathrm{A} / \mathrm{m}^{2}\right]$

J: Dimensionless electric current density

$J x_{\mathrm{bs}}$ : Dimensionless electric current density of the basic flow (along $x$-axis)

$J y_{\mathrm{bs}}$ : Dimensionless electric current density of the basic flow (along $y$-axis)

$J \widetilde{x}$ : Dimensionless electric current density of the amplitude (along $x$-axis)

$J \tilde{y}$ : Dimensionless electric current density of the amplitude (along $y$-axis)

$J \widetilde{z}:$ Dimensionless electric current density of the amplitude (along $z$-axis)

$k$ : $\quad$ Dimensionless wavenumber (along $z$-axis)

$l$ : $\quad$ Duct width in the $x$-axis [m]

$p$ : Pressure $[\mathrm{Pa}]$

$P: \quad$ Dimensionless pressure

$\widetilde{P}: \quad$ Dimensionless pressure of the amplitude

Pr: Prandtl number

$T_{0}$ : $\quad$ Reference temperature $[\mathrm{K}]$

$T: \quad$ Temperature $[\mathrm{K}]$ 
$\widetilde{U}:$ Dimensionless velocity of the amplitude (along $x$ axis)

v: Velocity vector $[\mathrm{m} / \mathrm{s}]$

V: Dimensionless velocity vector

$\widetilde{V}:$ Dimensionless velocity of the amplitude (along $y$ axis)

$W_{\mathrm{bs}}$ : Dimensionless velocity of the basic flow (along $z$ axis)

$\widetilde{W}:$ Dimensionless velocity of the amplitude (along $z$ axis)

$\alpha$ : Thermal diffusivity $\left[\mathrm{m}^{2} / \mathrm{s}\right]$

$\beta$ : Volumetric thermal expansion coefficient $\left[\mathrm{K}^{-1}\right]$

$\Theta_{\mathrm{bs}}$ : Dimensionless temperature of the basic flow

$\widetilde{\Theta}:$ Dimensionless temperature of the amplitude

$\lambda$ : Complex growth rate $[\mathrm{rad} / \mathrm{s}]$

$\nu$ : Kinematic viscosity $\left[\mathrm{m}^{2} / \mathrm{s}\right]$

$\rho_{0}: \quad$ Fluid density at reference temperature $\left[\mathrm{kg} / \mathrm{m}^{3}\right]$

$\sigma_{e}: \quad$ Electric conductivity $\left[\Omega^{-1} \mathrm{~m}^{-1}\right]$

$\phi: \quad$ Electric potential [V]

$\Phi_{\mathrm{bs}}$ : Dimensionless electric potential of the basic flow

$\widetilde{\Phi}$ : Dimensionless electric potential of the amplitude.

\section{Conflict of Interests}

The authors declare that there is no conflict of interests regarding the publication of this paper.

\section{References}

[1] Y. Shimomura, “The new ITER," Journal of Plasma and Fusion Research, vol. 79, no. 9, pp. 949-961, 2000.

[2] C. P. C. Wong, S. Malang, M. Sawan et al., "An overview of dual coolant $\mathrm{Pb}-17 \mathrm{Li}$ breeder first wall and blanket concept development for the US ITER-TBM design," Fusion Engineering and Design, vol. 81, no. 1-4, pp. 461-467, 2006.

[3] T. Tanaka, T. Muroga, T. Shikama et al., "Electrical insulating properties of ceramic coating materials for liquid Li blanket system under irradiation," Journal of Plasma and Fusion Research, vol. 83, no. 4, pp. 391-396, 2007.

[4] The society of Fluid Mechanics of Japan, Handbook of Fluid Mechanics (Japanese), The society of Fluid Mechanics of Japan, Tokyo, Japan, 2002.

[5] H. Ozoe and K. Okada, "The effect of the direction of the external magnetic field on the three-dimensional natural convection in a cubical enclosure," International Journal of Heat and Mass Transfer, vol. 32, no. 10, pp. 1939-1954, 1989.

[6] K. Okada and H. Ozoe, "Experimental heat transfer rates of natural convection of molten gallium suppressed under an external magnetic field in either the X, Y, or Z direction," Journal of Heat Transfer, vol. 114, no. 1, pp. 107-114, 1992.

[7] T. Tagawa and H. Ozoe, "Enhancement of heat transfer rate by application of a static magnetic field during natural convection of liquid metal in a cube," Journal of Heat Transfer, vol. 119, no. 2, pp. 265-271, 1997.

[8] T. Tagawa and H. Ozoe, "Enhanced heat transfer rate measured for natural convection in liquid gallium in a cubical enclosure under a static magnetic field," Journal of Heat Transfer, vol. 120, no. 4, pp. 1027-1032, 1998.
[9] G. Authié, T. Tagawa, and R. Moreau, "Buoyant flow in long vertical enclosures in the presence of a strong horizontal magnetic field. Part 2. Finite enclosures," European Journal of Mechanics, B/Fluids, vol. 22, no. 3, pp. 203-220, 2003.

[10] U. Burr, L. Barleon, P. Jochmann, and A. Tsinober, "Magnetohydrodynamic convection in a vertical slot with horizontal magnetic field," Journal of Fluid Mechanics, no. 475, pp. 21-40, 2003.

[11] C. Zhang, S. Eckert, and G. Gerbeth, "The flow structure of a bubble-driven liquid-metal jet in a horizontal magnetic field," Journal of Fluid Mechanics, vol. 575, pp. 57-82, 2007.

[12] K. Fujimura and M. Nagata, "Degenerate 1:2 steady state mode interaction-MHD flow in a vertical slot," Physica D: Nonlinear Phenomena, vol. 115, no. 3-4, pp. 377-400, 1998.

[13] T. Kakutani, "The hydromagnetic stability of the modified plane Couette flow in the presence of a transverse magnetic field," Journal of the Physical Society of Japan, vol. 19, no. 6, pp. 10411057, 1964.

[14] K. Fujimura and J. Mizushima, "Nonlinear equilibrium solutions for travelling waves in a free convection between vertical parallel plates," European Journal of Mechanics, B/Fluids, vol.10, no. 2, pp. 25-31, 1991.

[15] T. P. Lyubimova, D. V. Lyubimov, V. A. Morozov, R. V. Scuridin, H. B. Hadid, and D. Henry, "Stability of convection in a horizontal channel subjected to a longitudinal temperature gradient. Part 1. Effect of aspect ratio and Prandtl number," Journal of Fluid Mechanics, vol. 635, pp. 275-295, 2009.

[16] D. V. Lyubimov, T. P. Lyubimova, A. B. Perminov, D. Henry, and H. B. Hadid, "Stability of convection in a horizontal channel subjected to a longitudinal temperature gradient. Part 2. Effect of a magnetic field," Journal of Fluid Mechanics, vol. 635, pp. 297319, 2009. 

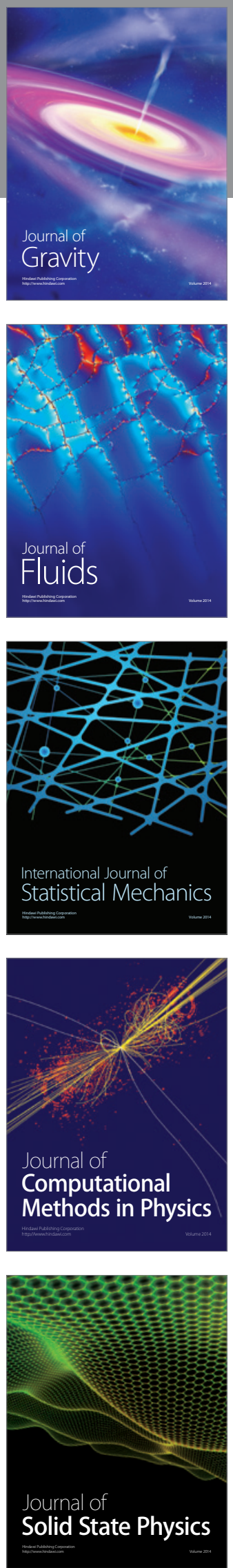

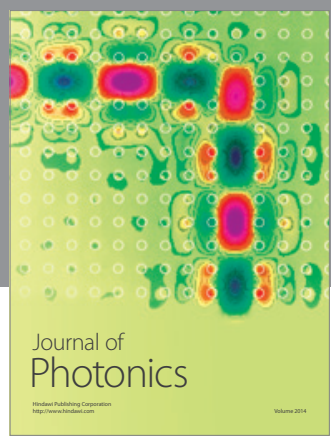

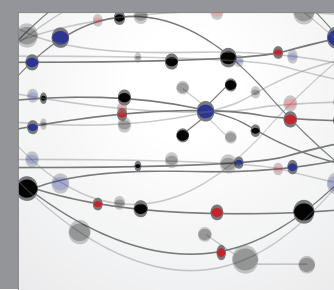

The Scientific World Journal

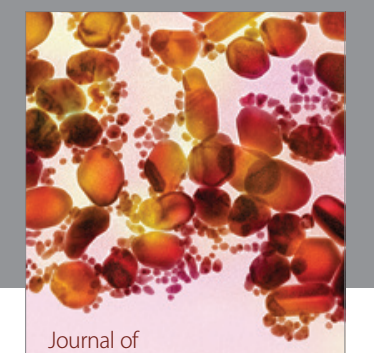

Soft Matter
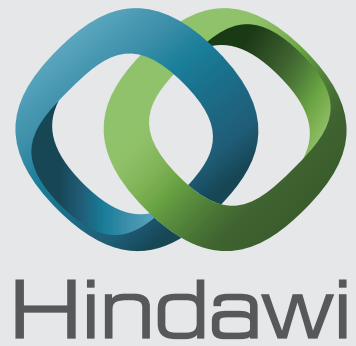

Submit your manuscripts at

http://www.hindawi.com
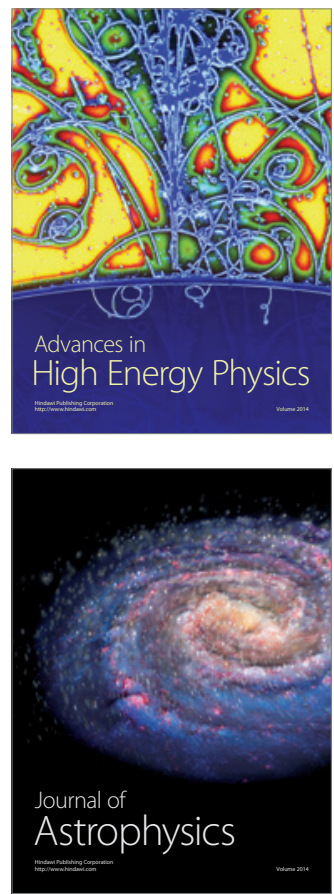
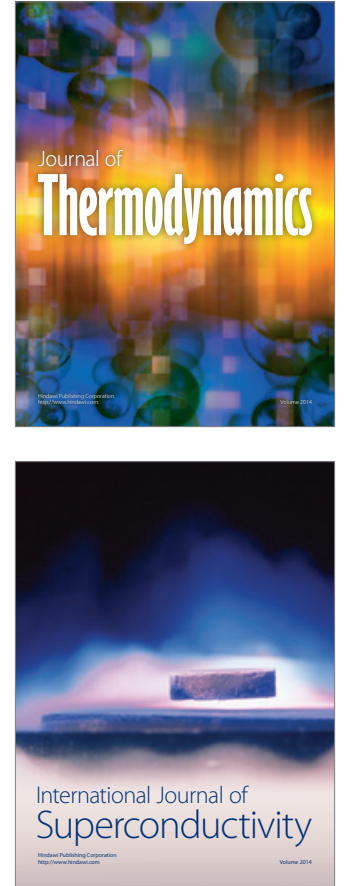
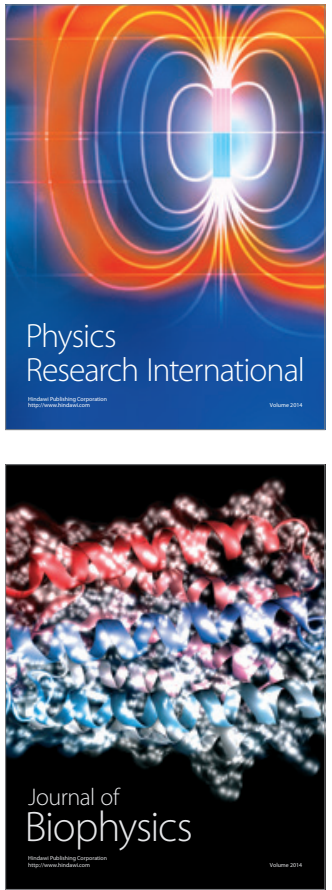
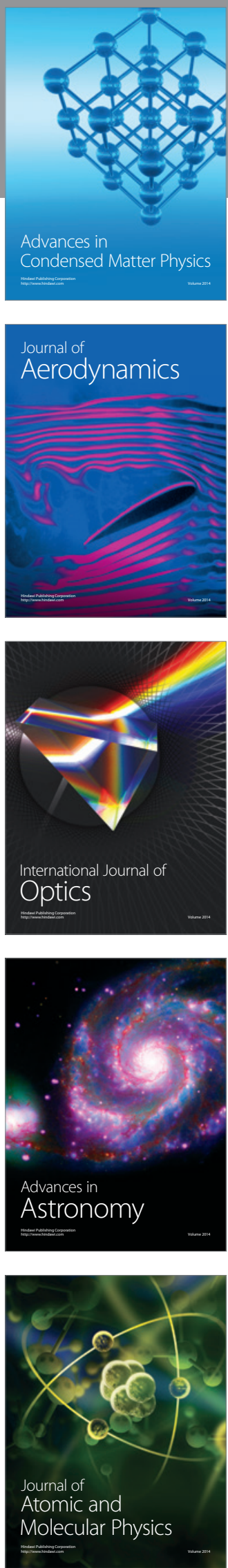